\title{
A valence bond description of the bromine halogen bond
}

\section{Davide Franchini, ${ }^{1}$ Alessandro Genoni, ${ }^{3 *}$ Federico Dapiaggi, ${ }^{1}$ Stefano Pieraccini, ${ }^{1,2 *}$ and Maurizio Sironi ${ }^{1,2^{*}}$}

Correspondence to: Maurizio Sironi (E-mail: maurizio.sironi@unimi.it)

\author{
${ }^{1}$ Davide Franchini, Federico Dapiaggi, Stefano Pieraccini, and Maurizio Sironi \\ Dipartimento di Chimica and INSTM UdR, Università degli Studi di Milano \\ Via Golgi 19, 20133, Milano (Italy) \\ ${ }^{2}$ Stefano Pieraccini, and Maurizio Sironi \\ CNR-ISTM (Istituto di Scienze e Tecnologie Molecolari del CNR) and INSTM UdR \\ Via Golgi 19, 20133, Milano (Italy) \\ ${ }^{3}$ Alessandro Genoni \\ Université de Lorraine \& CNRS, Laboratoire de Physique et Chimie Théoriques (LPCT), UMR CNRS 7019, 1 Boulevard \\ Arago, F-57078 Metz, France.
}

\begin{abstract}
A theoretical investigation on the nature of the halogen bond through a Valence-Bond approach has been carried out with two main goals: i) finding further confirmations of already existing explanations on the physical origins of the halogen bond and ii) possibly enriching the current models with new details. To achieve these goals we have exploited the spin-coupled method and we have performed computations on $\mathrm{RBr} \cdots \mathrm{NH} 3$ dimers characterized by a different electron withdrawing power of substituent $-R$ to the bromine atom. The analysis of typical spin-coupled descriptors (e.g., shapes and overlaps of the spin-coupled orbitals, weights of the spin-coupled structures) in the different cases and in function of the distance between the monomers allowed us to draw qualitative conclusions about the formation and the strength of the halogen bonds. In particular, the investigation not only confirmed the validity of already existing models (i.e., $\sigma$-hole and lump-hole models) but also highlighted interesting new features, such as the fact that the depletion of electron density around the bromine atom does not extend only towards the acceptor of the halogen bond, but also in the opposite direction (towards the substituent of the halogen), thus forming a sort of $\sigma$-tunnel, rather than a simple $\sigma$-hole.
\end{abstract}

\section{Introduction}

Halogen bonding $(X B)$ is an interesting noncovalent interaction ${ }^{1,2}$ that establishes between a halogen atom $X$ (covalently bonded to a substituent group R) and an acceptor group $B$ characterized by a nucleophilic character, following the general scheme $R-X \cdots B$. There are several factors that contribute to determine the strength of this peculiar bonding interaction. The most important ones are i) the electron withdrawing power of the substituent group $\mathrm{R}$, ii) the polarizability of the halogen atom $(\mathrm{I}>\mathrm{Br}>$ $\mathrm{Cl} \gg \mathrm{F}$ ) and, finally, iii) the basicity of the acceptor group $B$.
Despite its peculiar nature, basically consisting in a halogen atom acting as electrophile, XB interaction has been only barely studied in the past and it has become a hot topic only quite recently. In fact, also as a consequence of more and more detailed investigations that allowed us to gain fundamental insights into the nature of the interaction, several research groups have already envisaged the possibility of exploiting halogen bonding for functional applications.

Among them we can mention applications in medicinal chemistry and, particularly, in the rational design of new drugs. In fact, experimental evidences have interestingly shown that halogen bonds are able to efficiently stabilize protein-ligand complexes ${ }^{3-5}$ 
and to compete with hydrogen bonds in stabilizing DNA junctions through brominated uracil-bases $^{6}$. The importance of these observations is further enhanced by the fact that halogenated drugs are generally characterized by increased half-lives and better membrane permeability, which really makes halogen bond a fundamental key-interaction in modern rational drug design.

Moreover, since $X B$ interactions have been shown very efficient in tuning material properties (e.g., optical or magnetic properties) $)^{7,8}$ and in directing supramolecular assemblies $^{9-12}$, two important and related fields where halogen bond currently plays a crucial role are Materials Science and Crystal Engineering. ${ }^{13,14}$

As mentioned above, several efforts have been recently made i) to propose models with the aim of shedding further light on the nature of halogen bonding and ii) to develop new useful, theoretical/computational tools to correctly predict properties of halogen bond-based systems. For example, in the latter case, the increasing number of $\mathrm{X}$-ray resolved protein structures with halogenated ligands led to the need of developing an efficient strategy to describe halogen bonds by means of classical force field methods. ${ }^{15,16}$

Concerning the different models for halogen bonding that have been proposed over the years, the one introduced by Politzer ${ }^{17}$ could be probably considered as a milestone in this research area. In fact, it has been the first one to successfully and rationally describe the reason why commonly considered "negative atoms" as halogens interact with nucleophiles. According to this model, the establishment of a covalent $\mathrm{R}-\mathrm{X}$ bond causes a depletion of electron density in the outward region of the halogen atom along the $\mathrm{R}-\mathrm{X}$ bond direction (conventionally denoted as the z-axis throughout the paper), thus giving rise to a region of positive electrostatic potential (ESP), which is commonly called $\sigma$-hole, and a belt of negative ESP around this $\sigma$-hole to compensate it. This anisotropy of the ESP is the reason why halogens can interact with nucleophiles.
Moreover, the model explains i) the trend in the strength of halogen bonding, which is simply associated with the maximum value of the ESP in the $\sigma$-hole (for a given acceptor), and ii) the great directionality of the XB interaction, which is strictly related to the narrow localization of the $\sigma$-hole around the z-axis.

Another complementary model to the previous one is the lump-hole model ${ }^{18}$ that relies on the physical interpretation of the Laplacian of the electron density, $\nabla^{2} \rho(r)$. Following Bader's Quantum Theory of Atoms in Molecules, ${ }^{19}$ $\nabla^{2} \rho(r)$ can be used to distinguish between regions characterized by concentration $\left(\nabla^{2} \rho(r)\right.$ $<0)$ and depletion of electron density $\left(\nabla^{2} \rho(r)>\right.$ $0)$. Therefore, following this interpretation, in case of an XB interaction there will be an electron density depletion region $\left(\nabla^{2} \rho(r)>0\right)$, the hole, in proximity of the halogen atom and an electron density concentration region $\left(\nabla^{2} \rho(r)\right.$ $<0)$, the lump, localized on the acceptor. As the halogen atom approaches towards the acceptor, the hole will interact with the lump in a key-lock mechanism, thus leading to the establishment of the interaction.

All the previous models have been fully confirmed by accurate experimental charge density studies $^{20}$ and by several theoretical calculations. ${ }^{21}$ Nevertheless, to the best of our knowledge, the computations have been almost exclusively performed in the framework of Molecular Orbital (MO)-based techniques. Valence Bond (VB) strategies have been rarely exploited in this context, ${ }^{22}$ although, due to their intrinsic "chemical nature", the latter could provide significant insights into the features of the halogen bonding interaction. In fact, in all VB techniques, molecular electronic structure is described in terms of orbitals that are mainly localized on atoms and that significantly overlap when bonding interactions occur, thus preserving the traditional chemical picture of bond as schematically depicted through the well-known Lewis molecular structures. On the contrary, this traditional description is completely lost in all those computational strategies based on MOs since, 
in general, the obtained orbitals are completely delocalized on the whole systems under exam. Several efforts have been made to recover traditional chemical concepts (e.g., bond- and lone-pairs) also from MO-based calculations. For example, it is worth mentioning the a posteriori methods, ${ }^{23-27}$ which allow the determination of Localized Molecular Orbitals (LMOs) as unitary transformation of canonical Hartree-Fock MOs, or the a priori techniques, ${ }^{28-}$ 46 which exploit user-defined and chemically meaningful localization schemes to compute MOs that are extremely localized on small molecular fragments (atoms, bonds or functional groups) and that are easily transferable from a molecule to another. ${ }^{47-53}$ Attempts of exploiting localized MOs in a Valence Bond way were also proposed ${ }^{54-57}$ but, notwithstanding all these efforts, pure VB approaches remain the closest theoretical methods to traditional chemical concepts, although they are more computationally expensive than those based on MOs.

In this paper, also following the example of McAllister et al., who tried to rationalize and get further insights into the nature of halogenbonds between halomethanes and rare gases in terms of localized molecular orbitals, ${ }^{58}$ we present one of the first Valence Bond-based investigations on the nature of halogen bond. Here it is worth noting that our study mainly aimed at using fully VB concepts both to qualitatively confirm the validity of wellestablished models for the halogen bonding and to seek additional insights into the physical origin of this interaction.

To accomplish this task we have decided to resort to a particular VB strategy: the spincoupled (SC) method, ${ }^{59-61}$ which will be briefly described in the Theory section and which has been also recently combined with the X-ray constrained wave function approach ${ }^{62,63}$ of quantum crystallography ${ }^{64,65}$, giving rise to the new XC-SC strategy ${ }^{66}$ that can be potentially used for the extraction of chemically meaningful information (e.g., resonance structure weights, electron correlation effects $^{67}$, etc.) from high-resolution X-ray diffraction data.

To investigate the nature of the $X B$ interaction, we have performed Spin-Coupled calculations on different $\mathrm{RBr} \cdots \mathrm{NH} 3$ dimers $(\mathrm{R}=-\mathrm{H}, \mathrm{HCC}-$, $\mathrm{CN})$. In this way it was possible to draw some conclusions about the formation and the strength of the halogen bond in the different cases in terms of spin-coupled orbitals, their overlaps and weights of the spin-coupled structures. Finally, the obtained results have been discussed in function of the Politzer and lump-hole models mentioned above, showing that also VB calculations fully confirm them.

The paper is organized as follows: after a brief overview of the spin-coupled technique, we will dedicate a section dedicated to describe the strategies used to investigate the formation and strength of the halogen bond in the RBr..NH3 dimers. Afterwards, we will present and discuss the obtained results and, finally we will draw our final conclusions trying to find a connection between the outcomes of our calculations and commonly accepted models for interpreting and rationalizing the halogen bonding interaction.

\section{Theory}

In the spin-coupled method, the wave function for a system of $\mathrm{N}$ electrons is written by using non-orthogonal singly occupied orbitals that can interact by overlapping between themselves. Due to the non-orthogonality and the single occupancy of the spin-coupled orbitals there is, almost always, more than one way for coupling the individual spin of the $\mathrm{N}$ electrons to obtain the total spin of the system. These different coupling possibilities correspond to different spin-coupled structures. In particular, it is possible to show that, in case of a system of $\mathrm{N}$ electrons with total spin $\mathrm{S}$, for each value $M^{68}$ of the projection of $S$, we can write $\mathrm{f}_{\mathrm{S}}^{\mathrm{N}}$ linearly independent spin-coupled structures (and spin-eigenfunctions), where $\mathrm{f}_{\mathrm{S}}^{\mathrm{N}}$ is defined as: 


$$
\mathrm{f}_{\mathrm{S}}^{\mathrm{N}}=\frac{(2 \mathrm{~S}+1) \mathrm{N} !}{\left(\frac{1}{2} \mathrm{~N}+\mathrm{S}+1\right) !\left(\frac{1}{2} \mathrm{~N}-\mathrm{S}\right) !}
$$

Consequently, by associating each possible spincoupled structure with a particular $\mathrm{N}$-electron function $\psi_{\mathrm{S}, \mathrm{M} ; \mathrm{k}}^{\mathrm{N}}$, the global SC wave function for a system of $\mathrm{N}$ electrons in a spin-state $(\mathrm{S}, \mathrm{M})$ can be expressed as follows:

$$
\begin{aligned}
\psi_{\mathrm{S} C}^{\mathrm{S}, \mathrm{M}}=\sum_{\mathrm{k}=1}^{\mathrm{f}_{\mathrm{S}}^{\mathrm{N}}} \mathrm{c}_{\mathrm{sk}} & \psi_{\mathrm{S}, \mathrm{M} ; \mathrm{k}}^{\mathrm{N}} \\
& =\sum_{\mathrm{k}=1}^{\mathrm{f}_{\mathrm{S}}^{\mathrm{N}}} \mathrm{c}_{\mathrm{Sk}} \mathcal{A}\left(\Phi \Theta_{\mathrm{S}, \mathrm{M} ; \mathrm{k}}^{\mathrm{N}}\right)
\end{aligned}
$$

where $\mathcal{A}$ is the usual antisymmetrizing operator, $\Theta_{\mathrm{S}, \mathrm{M} ; \mathrm{k}}^{\mathrm{N}}$ is the $\mathrm{k}$-th spin-eigenfunction for the $\mathrm{N}$-electron system in the spin-state $(\mathrm{S}, \mathrm{M}), \Phi$ is the product of $\mathrm{N}$ spatial functions $\left\{\phi_{i}\right\}_{i=1}^{N}$ (namely, the spin-coupled orbitals)

$$
\begin{aligned}
& \Phi\left(\mathbf{r}_{1}, \mathbf{r}_{2}, \ldots \mathbf{r}_{\mathrm{N}}\right) \\
& =\phi_{1}\left(\mathbf{r}_{1}\right) \phi_{2}\left(\mathbf{r}_{2}\right) \ldots \phi_{\mathrm{N}}\left(\mathbf{r}_{\mathrm{N}}\right)
\end{aligned}
$$

and $\mathrm{c}_{\mathrm{S}, \mathrm{k}}$ are the spin-coupling coefficients that are used to weight the importance of each spincoupled structure in the wave function $\psi_{\mathrm{SC}}^{\mathrm{S}, \mathrm{M}}$, also through the determination of the ChirgwinCoulson coefficients ${ }^{69}$ defined like this:

$$
w_{S, k}=\left|c_{S, k}\right|^{2}+\sum_{j \neq k}^{f_{S}^{N}} c_{S, k} c_{S, j} S_{k j}
$$

with $S_{\mathrm{kj}}$ as the overlap integral between the spin-coupled structures $\psi_{\mathrm{S}, \mathrm{M} ; \mathrm{K}}^{\mathrm{N}}$ and $\psi_{\mathrm{S}, \mathrm{M} ; \mathrm{j}}^{\mathrm{N}}$

It is worth noting that, to reduce the computational cost associated with the spincoupled calculations, it is also usually advisable to subdivide the $\mathrm{N}$ electrons of the systems into two groups: a subset of $2 \mathrm{~N}_{1}$ core electrons and a subset of $\mathrm{N}_{\mathrm{v}}$ valence electrons. The former can be described by frozen doubly occupied Molecular Orbitals previously obtained by means of a proper Hartree-Fock computation on the system under exam. Since Molecular Orbitals are generally delocalized on the whole molecule, they are usually localized to better decide which of them to freeze. The valence electrons are really described at spin-coupled level. Therefore, in these cases, the spincoupled wave function assumes this form:

$$
\begin{aligned}
& \psi_{\mathrm{SC}}^{\mathrm{S}, \mathrm{M}}=\sum_{\mathrm{k}=1}^{\mathrm{f}_{\mathrm{S}}^{\mathrm{N}_{\mathrm{v}}}} \mathrm{c}_{\mathrm{Sk}} \psi_{\mathrm{S}, \mathrm{M} ; \mathrm{k}}^{\mathrm{N}} \\
& =\sum_{\mathrm{k}=1}^{\mathrm{f}_{\mathrm{S}}^{\mathrm{N}_{\mathrm{v}}}} \mathrm{c}_{\mathrm{Sk}} \mathcal{A}\left(\phi_{1}^{\mathrm{c}} \bar{\phi}_{1}^{\mathrm{c}} \ldots \phi_{\mathrm{N}_{1}}^{\mathrm{c}} \bar{\phi}_{\mathrm{N}_{1}}^{\mathrm{c}} \Phi_{\mathrm{v}} \Theta_{\mathrm{S}, \mathrm{M} ; \mathrm{k}}^{\mathrm{N}_{\mathrm{v}}}\right)
\end{aligned}
$$

where $\mathrm{c}_{\mathrm{Sk}}$ and $\mathcal{A}$ have an analogous meaning to the one seen for equation (2), $\phi_{i}^{c}$ is a frozen "core spin-orbital" with spatial part $\phi_{\mathrm{i}}^{\mathrm{c}}$ and spin part $\alpha, \bar{\phi}_{i}^{c}$ is a frozen "core spin-orbital" with spatial part $\phi_{\mathrm{i}}^{\mathrm{c}}$ and spin part $\beta$. As already mentioned above, the spatial parts $\left\{\phi_{i}^{c}\right\}$ can be canonical Molecular Orbitals or localized Molecular Orbitals. Furthermore, $\Theta_{\mathrm{S}, \mathrm{M} ; \mathrm{k}}^{\mathrm{N}_{\mathrm{V}}}$ is the k-th spin-eigenfunction for the $\mathrm{N}_{\mathrm{v}}$ valence electrons in the spin-state $(\mathrm{S}, \mathrm{M})$, and $\Phi_{\mathrm{v}}$ is the product of the $\mathrm{N}_{\mathrm{v}}$ "valence spin-coupled orbitals", namely:

$$
\begin{aligned}
& \Phi_{\mathrm{v}}\left(\mathbf{r}_{1}, \mathbf{r}_{2}, \ldots \mathbf{r}_{\mathrm{N}_{\mathrm{v}}}\right) \\
& =\phi_{1}^{v}\left(\mathbf{r}_{1}\right) \phi_{2}^{v}\left(\mathbf{r}_{2}\right) \ldots \phi_{N_{v}}^{v}\left(\mathbf{r}_{\mathrm{N}_{\mathrm{v}}}\right)
\end{aligned}
$$

Due to the doubly occupancy of the core orbitals, the active SC orbitals can be assumed orthogonal to the core orbitals without losing generality. In order to easily accomplish this, the active SC orbitals are expanded over the set of MOs $\left\{\phi_{\mu}(\boldsymbol{r})\right\}_{\mu=\mathrm{N}_{1}+1}^{M}$ consisting of the remaining (localized or canonical) occupied Hartree-Fock Molecular Orbitals and of all the virtual Hartree-Fock Molecular Orbitals, i.e.:

$$
\phi_{i}^{v}(\mathbf{r}) \sum_{\mu=\mathrm{N}_{1}+1}^{M} \mathrm{C}_{\mu \mathrm{i}} \phi_{\mu}(\boldsymbol{r})
$$

where $M$ is the dimension of the adopted basis- 
set (namely, the number of atomic orbitals initially used to perform the preliminary Hartree-Fock calculation). In this way the active SC orbitals are described using the full basis-set with the only constraint to be orthogonal to the frozen core orbitals.

The coefficients $\left\{\mathrm{C}_{\mu \mathrm{i}}\right\}$ of the spin-coupled orbitals expansions are thus obtained together with the spin-coupling coefficients $\left\{c_{\text {S,k }}\right\}$ (see equations (2) and (5)) by variationally minimizing the following energy functional:

$$
\mathrm{W}\left[\left\{\mathrm{C}_{\mu \mathrm{i}}\right\},\left\{\mathrm{c}_{\mathrm{Sk}}\right\}\right]=\frac{\left\langle\psi_{\mathrm{SC}}^{\mathrm{S}, \mathrm{M}}|\widehat{\mathrm{H}}| \psi_{\mathrm{SC}}^{\mathrm{S}, \mathrm{M}}\right\rangle}{\left\langle\psi_{\mathrm{SC}}^{\mathrm{S}, \mathrm{M}} \mid \psi_{\mathrm{SC}}^{\mathrm{S}, \mathrm{M}}\right\rangle}
$$

with $\widehat{\mathrm{H}}$ as the traditional non-relativistic Hamiltonian operator for a system of $\mathrm{N}$ electrons.

\section{Methods}

To investigate formation and strength of the halogen bond in $\mathrm{RBr} \cdots \mathrm{NH} 3$ dimers, we have considered three different $-\mathrm{R}$ substituents for the bromine atom: $-\mathrm{H}, \mathrm{HCC}-$ (acetylene group) and - CN (cyano group), clearly characterized by an increasing electron withdrawing power and, consequently, responsible for stronger halogen bonds.

To monitor the formation of a halogen bonding interaction in the $\mathrm{RBr} \cdots \mathrm{NH} 3$ dimers, we carried out spin-coupled calculations at different geometries previously obtained through relaxed geometry scans at Restricted Hartree-Fock (RHF) / 6-31G(d,p) ${ }^{70}$ level in which we varied the $\mathrm{Br} \cdots \mathrm{N}$ distance (from $2.39 \AA$ to $7.79 \AA$ for

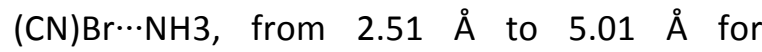
$\mathrm{HCCBr} \cdots \mathrm{NH} 3$ and from $2.58 \AA$ to $5.68 \AA$ for $\mathrm{HBr} \cdots \mathrm{NH} 3$ ) and in which we constrained the R$\mathrm{Br} \cdots \mathrm{N}$ angle equal to $180^{\circ}$. Finally, for all the three dimers we have also considered the corresponding asymptotic structures, basically corresponding to the two isolated monomers $\mathrm{RBr}$ and $\mathrm{NH} 3$ at very large distance ( $\mathrm{R}-\mathrm{Br} \cdots \mathrm{N}$ angle always constrained to $180^{\circ}$ ).

The obtained geometries for the $\mathrm{RBr} \cdots \mathrm{NH} 3$ dimers have been afterwards exploited to perform single point spin-coupled calculations with only 10 active electrons (corresponding to valence electrons in equations (5) and (6)). The remaining electrons (core electrons in equations (5) and (6)) were described through frozen doubly occupied Localized Molecular Orbitals) resulting from the application of the Pipek-Mezey localization technique to occupied Molecular Orbitals previously obtained through RHF calculations. These orbitals correspond to the frozen "core SC orbitals" in equation (5).

For this reason, in all our SC computations, only ten spin-coupled orbitals were directly optimized. They describe the three-bromine lone-pairs, the nitrogen lone-pair and the $\mathrm{R}-\mathrm{Br}$ bond electron-pair. As briefly mentioned in the Theory section, the "valence SC orbitals" have been obtained by fully expanding them, without any symmetry constraint, in the set of orbitals consisting of the remaining occupied PipekMezey LMOs (i.e., the non-frozen ones) and of the virtual (and completely delocalized) RHF MOs of the system under exam. So, for each SC orbital, the guess was a linear combination of the unfrozen occupied Pipek-Mezey LMOs and of the virtual RHF MOs. As mentioned above, it is worth stressing that, unlike the occupied Pipek-Mezey LMOs, the virtual molecular orbitals are completely delocalized on the investigated systems and, therefore, this allowed us to set up an educated and, at the same time, quite general guess without biasing the following spin-coupled calculations.

The 10 singly occupied spin-coupled orbitals (see Figures S1 and S2 for two- and threedimensional graphical representations of the SC

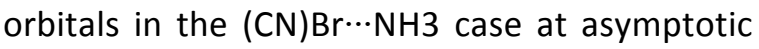
distance) resulting from our computations were afterwards classified, by observing their components expressed in atomic orbitals basis, and labeled in this way (always assuming the R$\mathrm{Br}$ bond axis as the $\mathrm{z}$-axis):

- $\phi_{1}$ : $\mathrm{sp}_{\mathrm{z}}$-like hybrid orbital mainly localized on the bromine atom and deformed toward the substituent $\mathrm{R}$. It is worth noting that this orbital is 
characterized by a hole that can be associated with the $\sigma$-hole of the Politzer model.

- $\phi_{2}$ : $\mathrm{sp}_{\mathrm{z}}$-like hybrid orbital mainly localized on the carbon atom $((\mathrm{CN}) \mathrm{Br}$ and $\mathrm{HCCBr}$ ) or the hydrogen atom $(\mathrm{HBr})$ and deformed toward the bromine atom.

- $\phi_{3}, \phi_{4}$ : orbitals resembling $\mathrm{sp}_{\mathrm{z}}$ hybrid lone-pair orbitals localized on the bromine atom, both of them characterized by a non-negligible $\sigma$ component. $\phi_{3}$ is more contracted along the z-axis, while $\phi_{4}$ is more spread towards the two directions perpendicular to the z-axis.

- $\phi_{5}, \phi_{6}$ : orbitals resembling $p_{y}$ lone-pair orbitals localized on the bromine atom. Both of them are characterized by a weak, but non-negligible, $\sigma$ component and by a small overlap with orbitals $\phi_{3}$ and $\phi_{4}$.

- $\phi_{7}, \phi_{8}$ : orbitals resembling $\mathrm{p}_{\mathrm{x}}$ lone-pair orbitals localized on the bromine atom. Both of them are characterized by a weak, but non-negligible, $\sigma$ component and by a small overlap with orbitals $\phi_{3}$ and $\phi_{4}$. They are symmetry related to orbitals $\phi_{5}$ and $\phi_{6}$.

- $\phi_{9}, \phi_{10}$ : orbitals describing the $p_{z}$ lonepair localized on the nitrogen atom. $\phi_{9}$ is more spread towards the direction of the bromine atom than orbital $\phi_{10}$.

Afterwards, they were analyzed by monitoring some of their overlaps and some of their square moduli differences, both in function of the $\mathrm{Br} \cdots \mathrm{N}$ distance and in function of the substituent group R. As we will explain in the Results section, this allowed us to detect partial delocalizations clearly associable with the presence of a halogen-bond interaction. Moreover, it is worth noting that, according to equation (1), the 10 active electrons (and consequently the 10 associated SC orbitals mentioned above) in a singlet state can be spincoupled in 42 different ways, which correspond to 42 different spin-coupled structures contributing to the global SC wave function (see Equation (5)). In order to have a direct connection with the traditional Lewis chemical structures, the Rumer spin eigenfunctions basis was adopted. ${ }^{71,72}$ The weights of all the structures have been also carefully monitored in function of the $\mathrm{Br} \cdots \mathrm{N}$ distance and in function of the substituent group $R$ to study the formation and the strength of the halogen-bond in the investigated systems.

To obtain the dimers geometries the Gaussian $09^{73}$ software has been adopted, while, for the SC calculations, a code developed in our research group has been used. ${ }^{74}$

\section{Results}

As mentioned in the previous section, different descriptors were considered to study the formation and the strength of the halogen bond in the investigated $\mathrm{RBr} \cdots \mathrm{NH} 3$ dimers: overlap between the SC orbitals, shape of the SC orbitals and weight of the spin-coupled structures.

At first, we mainly focused on the analysis of the overlap integrals between the 10 optimized SC orbitals already described in the previous section. In particular, since orbitals $\phi_{9}$ and $\phi_{10}$ are mainly localized on the nitrogen atom, we decided to monitor their overlaps with the other eight SC orbitals (mainly localized on $\mathrm{Br}$ ) in function of the distance, with the aim of possibly correlating their expansions towards the bromine atom with the formation of the halogen bond. From our computations, we have immediately observed that, as expected, for all the different $\mathrm{Br} \cdots \mathrm{N}$ distances, the largest overlaps of $\phi_{9}$ and $\phi_{10}$ (the orbitals describing the nitrogen lone-pair) were with z-symmetry orbitals $\left(\phi_{1}, \phi_{2}, \phi_{3}\right.$ and $\left.\phi_{4}\right)$, while the overlaps with the other SC orbitals were almost negligible. In particular, the greatest overlaps of $\phi_{9}$ and $\phi_{10}$ are with orbital $\phi_{1}$, which, from a 
Valence Bond point of view, could be the main responsible for the halogen bond interaction. In fact, as mentioned above and as it can be clearly seen in Figure S1, this orbital is characterized by a hole that can be easily associated with the the $\sigma$-hole on the $\mathrm{Br}$ atom. In Figure 1, we have also graphically depicted how the overlaps of $\phi_{9}$ (Figure 1A) and $\phi_{10}$ (Figure 1B) with $\phi_{1}, \phi_{2}, \phi_{3}$ and $\phi_{4}$ vary in function of the $\mathrm{Br} \cdots \mathrm{N}$ distance for the (CN) $\mathrm{Br} \cdots \mathrm{NH} 3$ dimer. It is easy to observe that, both for $\phi_{9}$ and for $\phi_{10}$, the overlaps increase as the two monomers approach, thus revealing the possible presence of a halogen bond interaction at shorter distances. Analogous trends have been also noted for the $\mathrm{HCCBr} \cdots \mathrm{NH} 3$ and $\mathrm{HBr} \cdots \mathrm{NH} 3$ dimers (see Figures

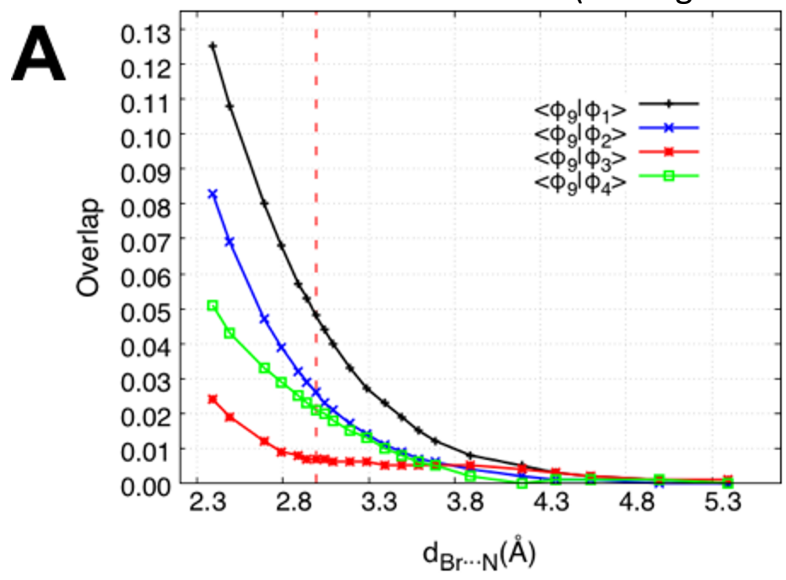

S3 and S4 in the Supplementary Material), although it is immediately evident that the overlaps magnitudes decrease when substituents $\mathrm{HCC}-$ and $-\mathrm{H}$ are taken into account. This can be also evinced from Table 1, where we have reported the overlaps at the equilibrium distances for the three investigated systems, which confirms that the strength of the halogen bond decreases as the electronwithdrawing character of the $R$ substituent reduces.

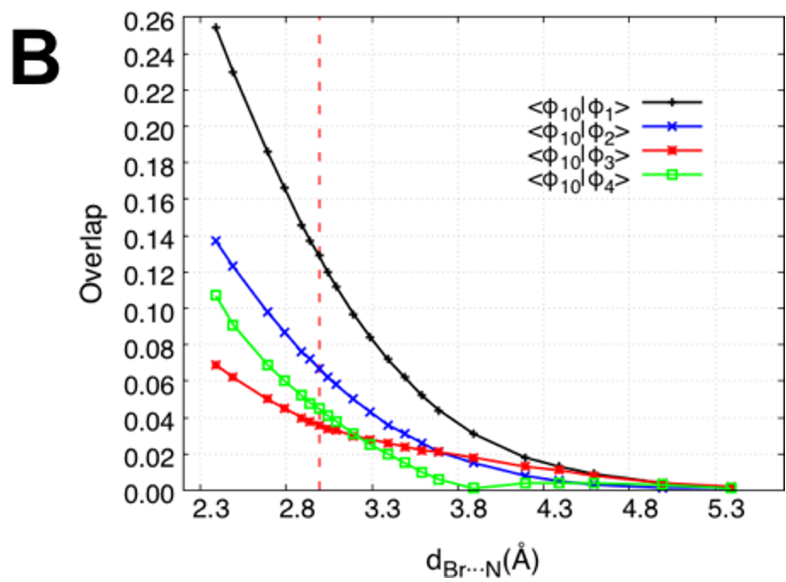

Figure 1. Overlaps of the spin-coupled orbitals $\phi_{9}$ and $\phi_{10}$ with the spin-coupled orbitals $\phi_{1}, \phi_{2}, \phi_{3}$ and $\phi_{4}$ in function of the $\mathrm{Br} \cdots \mathrm{N}$ distance for the $(\mathrm{CN}) \mathrm{Br} \cdots \mathrm{NH} 3$ dimer. The vertical red dotted lines indicate the $\mathrm{Br} \cdots \mathrm{N}$ equilibrium distance. 
Table 1. Absolute values of the overlaps of the spin-coupled orbitals $\phi_{9}$ and $\phi_{10}$ with the spin-coupled orbitals $\phi_{1}, \phi_{2}, \phi_{3}$ and $\phi_{4}$ at the equilibrium distances for the dimers (CN) $\mathrm{Br} \cdots \mathrm{NH} 3, \mathrm{HCCBr} \cdots \mathrm{NH} 3$ and $\mathrm{HBr} \cdots \mathrm{NH} 3$

\begin{tabular}{|c|c|c|c|c|c|c|}
\hline & \multicolumn{2}{|c|}{$(\mathrm{CN}) \mathrm{Br} \cdots \mathrm{NH}_{3}$} & \multicolumn{2}{|c|}{$\mathrm{HCCBr} \cdots \mathrm{NH}_{3}$} & \multicolumn{2}{|c|}{$\mathrm{HBr} \cdots \mathrm{NH}_{3}$} \\
\hline & $\phi_{9}$ & $\phi_{10}$ & $\phi_{9}$ & $\phi_{10}$ & $\phi_{9}$ & $\phi_{10}$ \\
\hline$\phi_{1}$ & 0.048 & 0.129 & 0.037 & 0.103 & 0.028 & 0.085 \\
\hline$\phi_{2}$ & 0.026 & 0.067 & 0.019 & 0.054 & 0.015 & 0.047 \\
\hline$\phi_{3}$ & 0.021 & 0.045 & 0.008 & 0.035 & 0.010 & 0.023 \\
\hline$\phi_{4}$ & 0.007 & 0.036 & 0.015 & 0.029 & 0.008 & 0.028 \\
\hline
\end{tabular}

A second descriptor to reveal the presence of an $X B$ interaction in the examined systems was the shape of the obtained orbitals. At first, we have considered orbitals $\phi_{9}$ and $\phi_{10}$, which are mainly localized on the nitrogen atom. For each of them, we have evaluated and plotted the difference between their squared moduli at the equilibrium and asymptotic distances (i.e., $\left|\phi_{9}^{\mathrm{eq}}\right|^{2}-\left|\phi_{9}^{\infty}\right|^{2}$ and $\left.\left|\phi_{10}^{\mathrm{eq}}\right|^{2}-\left|\phi_{10}^{\infty}\right|^{2}\right)$. The

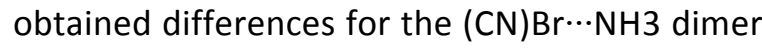
are depicted in Figures $2 \mathrm{~A}$ and $2 \mathrm{~B}$, where we can easily observe that both orbitals $\phi_{9}$ and $\phi_{10}$ are more localized on the nitrogen atom at the asymptotic distance, while they clearly shift towards the bromine atom at the equilibrium distance. Moreover, the effect is much more evident for orbital $\phi_{10}$ than for orbital $\phi_{9}$. Also these observations can be considered as another clear evidence of the formation of a halogen bond interaction $R X \cdots B$ at the equilibrium distance and can be rationalized though a "pictorial" chemical representation in which one electron of the lone-pair localized on acceptor atom B "moves" towards electrophilic region $X$, while the other electron of the pair remains on the nucleophilic site. Furthermore, it is quite interesting and unexpected that, during the formation of the halogen bond, the spin-coupled orbital $\left(\phi_{10}\right)$ describing the electron donated by acceptor B (in this case the nitrogen atom), also delocalizes over the region of the $\mathrm{C}-\mathrm{Br}$ bond and not only outward this bond (see Figures 2B, 2D and 2F). This can be interpreted (from a valence-bond point of view) as if the $\sigma$-hole, which is usually observed along the z-axis, in the region outward the halogen atom, existed also behind the halogen in the direction of the substituent group $R$. The calculation of the RHF/6-31G(d,p) electrostatic potential in the $x z$ plane indeed shows (see Figure 3 ) that the depletion of electron density along the z-axis around the halogen atom is not only localized outward the halogen atom, but it can be also seen as an extended positive region of electrostatic potential along the $\mathrm{R}-\mathrm{Br}$ bond. Therefore, the $\sigma$-hole could be actually seen as a " $\sigma$-tunnel" in terms of spin-coupled orbitals. This positive region of electrostatic potential attracts negative electron density from acceptor $B$ and this is probably the reason why, in all our spin-coupled calculations performed at the equilibrium distances, the largest overlaps for the "shifted" (see Figure 2 ) orbitals $\phi_{9}$ and $\phi_{10}$ have been observed with orbital $\phi_{1}$ (see Figure $1)$, namely the orbital characterized by a hole on the $\mathrm{Br}$ atom ascribable to the $\sigma$-hole of the Politzer model .

For the sake of completeness, in Figure 2 we have also reported the differences between the 
squared moduli of orbitals $\phi_{9}$ and $\phi_{10}$ at the equilibrium and asymptotic distances for the dimers $\mathrm{HCCBr} \cdots \mathrm{NH} 3$ (Figures $2 \mathrm{C}$ and 2D) and $\mathrm{HBr} \cdots \mathrm{NH} 3$ (Figures $2 \mathrm{E}$ and $2 \mathrm{~F}$ ). Although of lower extent, the trends are analogous to those observed for (CN)Br..NH3, further indicating that the strength of the $X B$ interaction reduces when a less electron-withdrawing group is bonded to the halogen atom.
A

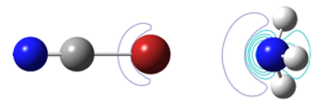

C

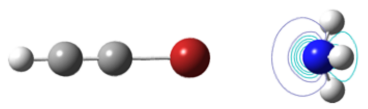

E

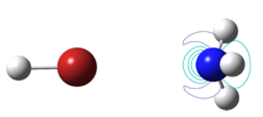

B

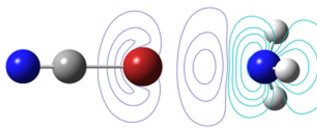

D

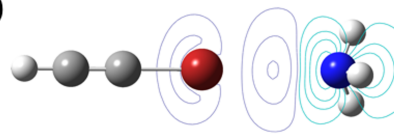

$\mathbf{F}$

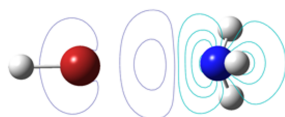

Figure 2. Differences between the squared moduli of the spin-coupled orbitals $\phi_{9}(A, C$ and $E)$ and $\phi_{10}(B, D$ and $F)$ at the

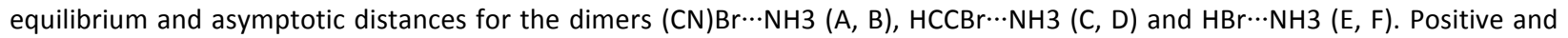
Negative contour levels are depicted in purple and light blue, respectively. The absolute values (in au) of the positive and negative contours increase in steps of $2 \times 10^{\mathrm{n}}, 4 \times 10^{\mathrm{n}}$ and $8 \times 10^{\mathrm{n}}$, with $\mathrm{n}$ ranging from -3 to 0 and increasing by 1 at every step. The contours levels of $5 \times 10^{-4}$ au and $1 \times 10^{-4}$ au have been added for the sake of completeness, both for the negative and positive contours.

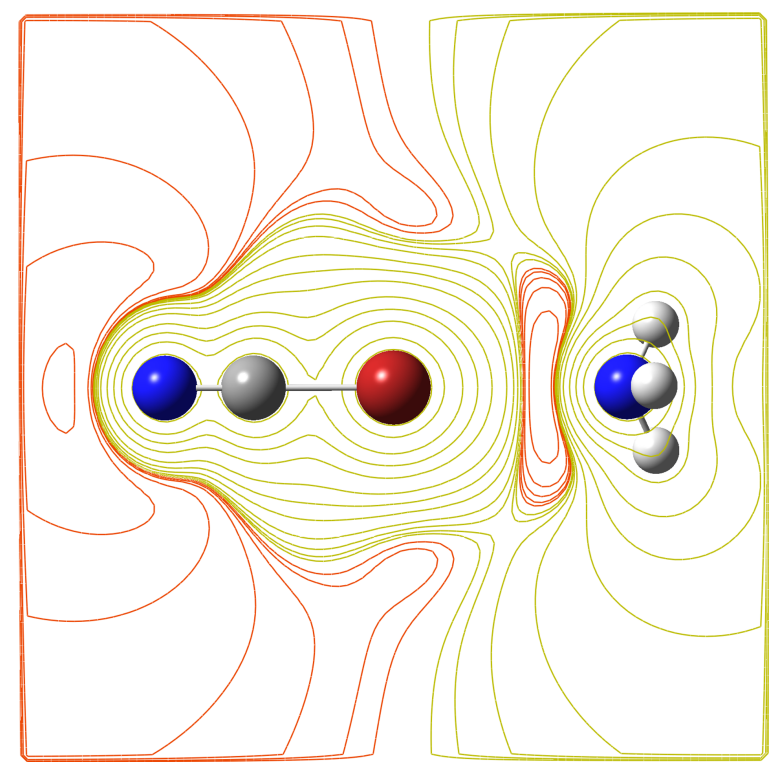

Figure 3. Contour levels of the Electrostatic Potential in the $\mathrm{xz}$ plane for the $(\mathrm{CN}) \mathrm{Br} \cdots \mathrm{NH} 3$ dimer. Positive and Negative contour levels are depicted in yellow and orange, respectively. The absolute values (in au) of the positive and negative contours increase in steps of $2 \times 10^{n}, 4 \times 10^{n}$ and $8 \times 10^{n}$, with $n$ ranging from -3 to 0 and increasing by 1 at every step. The contours levels of $5 \times 10^{-4}$ au and $1 \times 10^{-4}$ au have been added for the sake of completeness, both for the negative and positive contours. 
Finally, we have considered the $\mathrm{Br}$-centered and z-symmetry orbitals $\phi_{1}, \phi_{3}$ and $\phi_{4}$. Also in this case, we have evaluated and plotted the differences between their squared moduli at the equilibrium and asymptotic geometries. The

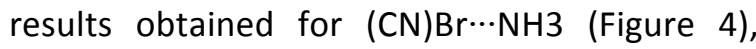
$\mathrm{HCCBr} \cdots \mathrm{NH} 3$ (Figue 5) and $\mathrm{HBr} \cdots \mathrm{NH} 3$ (Figure 6) are completely analogous and show that, at the equilibrium distance, the three examined spincoupled orbitals shift/delocalize towards the substituent group R. This leaves a depletion of charge around the bromine atom that can be easily associated with the $\sigma$-hole of the Politzer model. Hence, this is another evidence of the existence of halogen bonds when we are at the equilibrium geometry. However, the entity of the orbitals delocalizations does not change significantly among the different dimers and, therefore, in this case, from Figures 4, 5 and 6 we cannot infer on the strength of the different halogen bonds.
A

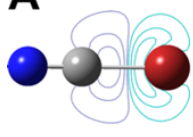

B
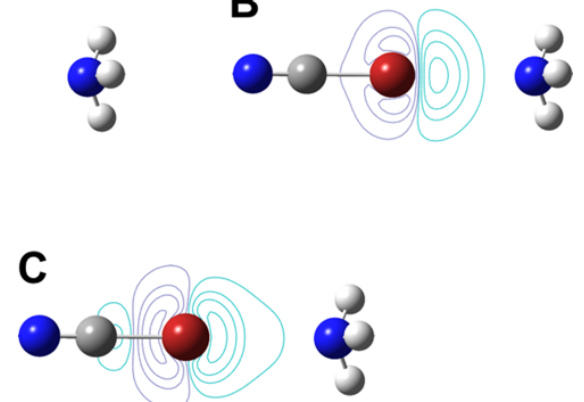

Figure 4. Differences between the squared moduli of the spin-coupled orbitals $\phi_{1}(\mathrm{~A}), \phi_{3}$ (B) and $\phi_{4}$ (C) at the equilibrium and asymptotic distances for the (CN) $\mathrm{Br} \cdots \mathrm{NH} 3$ dimer. Positive and Negative contour levels are depicted in purple and light blue, respectively. The absolute values (in au) of the positive and negative contours increase in steps of $2 \times 10^{n}, 4 \times 10^{n}$ and $8 \times 10^{n}$, with $n$ ranging from -3 to 0 and increasing by 1 at every step. The contours levels of 5 $\times 10^{-4}$ au and $1 \times 10^{-4}$ au have been added for the sake of completeness, both for the negative and positive contours.
A

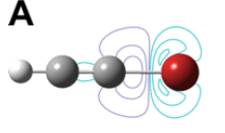

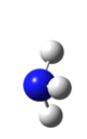

$c$

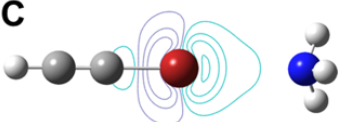

B

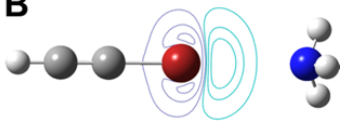

Figure 5. Differences between the squared moduli of the spin-coupled orbitals $\phi_{1}(\mathrm{~A}), \phi_{3}(\mathrm{~B})$ and $\phi_{4}(\mathrm{C})$ at the equilibrium and asymptotic distances for the $\mathrm{HCCBr} \cdots \mathrm{NH} 3$ dimer. Positive and Negative contour levels are depicted in purple and light blue, respectively. The absolute values (in au) of the positive and negative contours increase in steps of $2 \times 10^{n}, 4 \times 10^{n}$ and $8 \times 10^{n}$, with $n$ ranging from -3 to 0 and increasing by 1 at every step. The contours levels of 5 $\times 10^{-4}$ au and $1 \times 10^{-4}$ au have been added for the sake of completeness, both for the negative and positive contours 
A

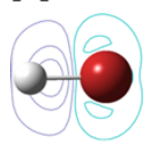

B

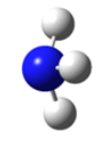

C

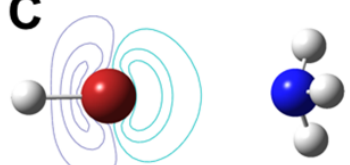

Figure 6. Differences between the squared moduli of the spin-coupled orbitals $\phi_{1}(\mathrm{~A}), \phi_{3}(\mathrm{~B})$ and $\phi_{4}(\mathrm{C})$ at the equilibrium and asymptotic distances for the $\mathrm{HBr} \cdots \mathrm{NH} 3$ dimer. Positive and Negative contour levels are depicted in purple and light blue, respectively. The absolute values (in $\mathrm{au}$ ) of the positive and negative contours increase in steps of $2 \times 10^{n}, 4 \times 10^{n}$ and $8 \times 10^{n}$, with $n$ ranging from -3 to 0 and increasing by 1 at every step. The contours levels of 5 $\times 10^{-4}$ au and $1 \times 10^{-4}$ au au have been added for the sake of completeness, both for the negative and positive contours.

As mentioned above, to complete our Valence Bond investigation of the halogen bond interaction, we have also decided to consider the weights of the different SC structures that contribute to the global spin-coupled wave functions. In particular, we have decided to monitor how the absolute values of the Chirgwin-Coulson coefficients associated with the different SC structures vary in function of the $\mathrm{Br} \cdots \mathrm{N}$ distance in the different cases.

We have already pointed out that the 10 active electrons of our singlet-state systems can be potentially spin-coupled in 42 different ways corresponding to 42 spin-coupled structures in wave function (5). However, in the range of the $\mathrm{Br} \cdots \mathrm{N}$ distances examined with our calculations, we have observed that only 5 structures are characterized by Chirgwin-Coulson coefficients significantly different from zero. Therefore, we considered them as the predominant SC structures for our systems and they were the only ones for which we have monitored the variation of the weight in function of the $\mathrm{Br} \cdots \mathrm{N}$ distance. They are: i) the perfect pairing structure, namely the structure corresponding to spin-coupling $\phi_{1}-\phi_{2} \phi_{3}-\phi_{4} \phi_{5}-$ $\phi_{6} \quad \phi_{7}-\phi_{8} \quad \phi_{9}-\phi_{10}$ (from now on

indicated as structure 1); ii) structure 2, corresponding to spin-coupling $\phi_{1}-\phi_{4} \phi_{2}-$ $\phi_{3} \phi_{5}-\phi_{6} \phi_{7}-\phi_{8} \phi_{9}-\phi_{10}$; iii) structure 3 , corresponding to spin-coupling $\phi_{1}-$ $\phi_{9} \phi_{2}-\phi_{10} \phi_{3}-\phi_{4} \phi_{5}-\phi_{6} \phi_{7}-\phi_{8}$; iv) structure 4 , corresponding to spin-coupling $\phi_{1}-\phi_{4} \phi_{2}-\phi_{10} \phi_{3}-\phi_{9} \phi_{5}-\phi_{6} \phi_{7}-$ $\left.\phi_{8} ; v\right)$ structure 5 , corresponding to spincoupling $\phi_{1}-\phi_{2} \phi_{3}-\phi_{9} \phi_{4}-\phi_{10} \phi_{5}-$ $\left.\phi_{6} \phi_{7}-\phi_{8}\right)$.

The first two structures correspond to the predominant ones at the asymptotic distances (i.e., for the isolated monomers), while structures 3, 4 and 5 are actually the ones that, from a traditional chemical point of view, can be easily associated with the formation of the halogen bonding interaction. In fact, each of them involves the pairing of a SC orbital localized on the nitrogen atom with a zsymmetry SC orbital localized on the bromine atom.

Although quite small, for all the investigated dimers, the Chirgwin-Coulson coefficients of structures 3-5 consistently increase as the two monomers approach and become significantly different from zero around the equilibrium distance (see Figure 7 for the $(\mathrm{CN}) \mathrm{Br} \cdots \mathrm{NH} 3$ dimer and Figures $\mathrm{S} 5$ and $\mathrm{S} 6$ in the Supplementary Material for the $\mathrm{HCCBr} \cdots \mathrm{NH} 3$ and the $\mathrm{HBr} \cdots \mathrm{NH} 3$ dimers, respectively). Therefore, considering the "chemical meaning" of structures 3-5 discussed above, we believe that this is a further indication of the existence of a halogen bond for the different systems at their equilibrium geometries. Furthermore, also in this case, we have clear evidences that the strength of the $X B$ interaction increases with the electron-withdrawing power of the substituent group $R$. In fact, if we consider the weights of structures $3-5$ at the $\mathrm{Br} \cdots \mathrm{N}$ equilibrium distances (see Table 2), we can easily note that the largest and lowest values are observed for $\mathrm{R}=-\mathrm{CN}$ and $\mathrm{R}=-\mathrm{H}$, respectively, which confirms the predicted trend according to the $\sigma$-hole model proposed by Politzer. 
Structure 3

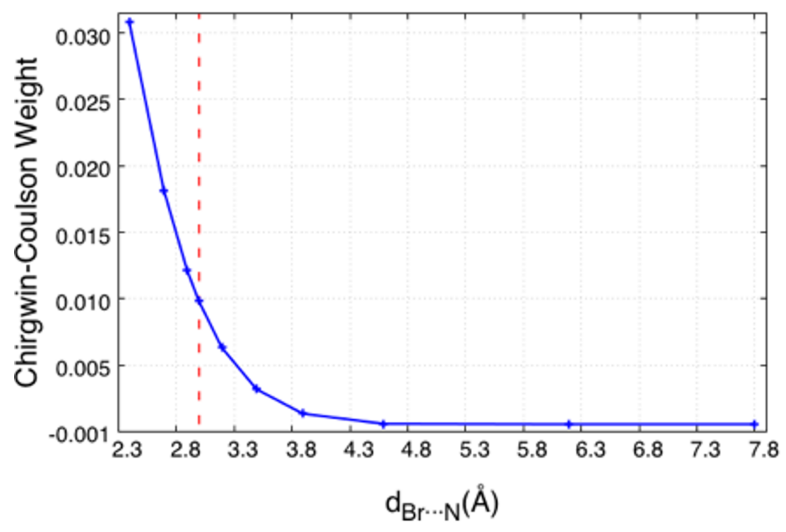

Structure 4

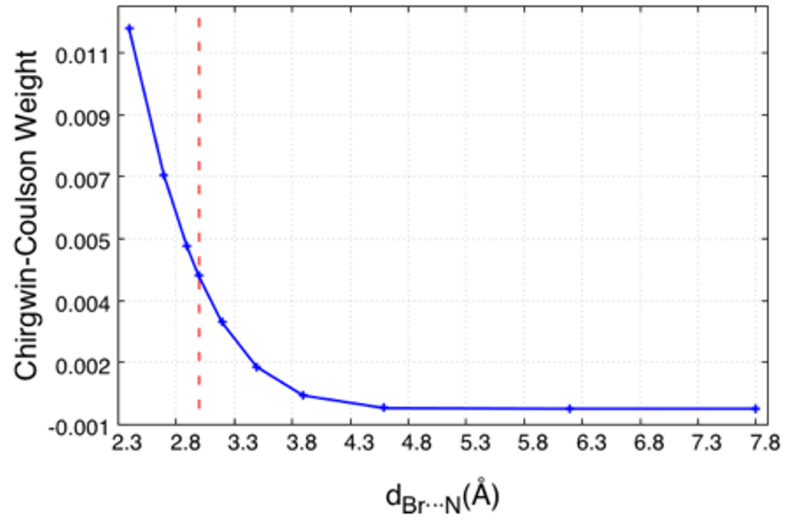

\section{Structure 5}

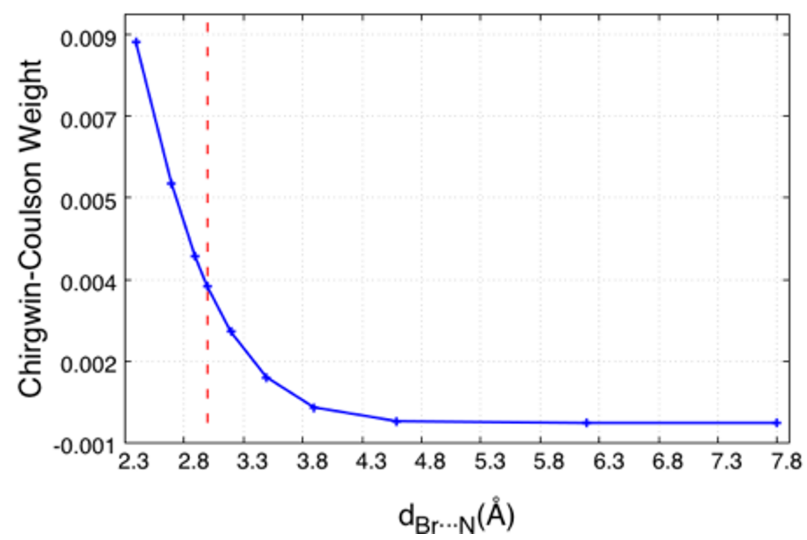

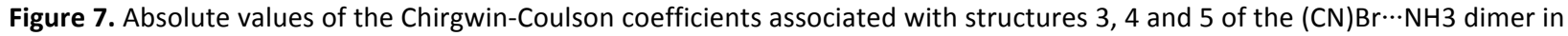
function of the $\mathrm{Br} \cdots \mathrm{N}$ distance. The vertical red dotted lines indicate the $\mathrm{Br} \cdots \mathrm{N}$ equilibrium distance.

Table 2. Absolute values of the Chirgwin-Coulson weights for spin-coupled structures 3,4 and 5 of dimers $(\mathrm{CN}) \mathrm{Br} \cdots \mathrm{NH} 3, \mathrm{HCCBr} \cdots$ $\mathrm{NH} 3$ and $\mathrm{HBr} \cdots \mathrm{NH} 3$ at their $\mathrm{Br} \cdots \mathrm{N}$ equilibrium distances.

\begin{tabular}{|llll|}
\hline & $(\mathrm{CN}) \mathrm{Br} \cdots \mathrm{NH} 3$ & $\mathrm{HCCBr} \cdots \mathrm{NH} 3$ & $\mathrm{HBr} \cdots \mathrm{NH} 3$ \\
\hline Structure 3 & $9.35 \times 10-3$ & $6.82 \times 10-3$ & $4.93 \times 10-3$ \\
Structure 4 & $4.30 \times 10-3$ & $3.31 \times 10-3$ & $2.42 \times 10-3$ \\
Structure 5 & $3.34 \times 10-3$ & $2.04 \times 10-3$ & $7.9 \times 10-4$ \\
\hline
\end{tabular}

\section{Conclusions}

In this paper we have performed a ValenceBond study of the halogen bond interaction exploiting the spin-coupled method and using the dimers (CN) $\mathrm{Br} \cdots \mathrm{NH} 3, \mathrm{HCCBr} \cdots \mathrm{NH} 3$ and $\mathrm{HBr} \cdots \mathrm{NH} 3$ as case studies. In particular, to investigate the nature and the strengths of the $\mathrm{Br} \cdots \mathrm{N}$ interaction in the different systems, we have considered three different descriptors associated with the spin-coupled technique: the overlap between the spin-coupled orbitals, the shapes of these orbitals and the ChirgwinCoulson weights of the spin-coupled structures. 
All these descriptors have not only shown that a $\mathrm{Br} \cdot \mathrm{N}$ interaction actually exists when the $\mathrm{RBr}$ and NH3 monomers approach, but they were also able to confirm the expected trend for the strength of the interaction, as predicted by the Politzer and the lump-hole models for halogen bond.

Analyzing the overlaps and the shapes of the spin-coupled orbitals, we were also able to qualitatively confirm that, in presence of a halogen bonding interaction, a depletion of electron density occurs around the halogen atom (bromine in our case) with a consequent shift/delocalization of the electron density from the acceptor (nitrogen in our case) to the halogen. In other words, our spin-coupled calculations confirmed the mechanism of interaction described by the Politzer and lumphole models (e.g. o-hole and depletion of electron density around the halogen atom).

Moreover, analyzing our results we have also interestingly observed that, as a result of the halogen bond formation, the spin-coupled orbital describing one of the electrons of the donor's lone pair is localized on the halogen atom in the direction pointing towards the substituent group $\mathrm{R}$ of the halogen so that the $\sigma$-hole becomes a $\sigma$-tunnel for the spin-coupled orbitals. This agrees with simple calculations of electrostatic potentials that we have performed on the systems under exam.

Finally, it is worth pointing out again that the paper has reported only a qualitative investigation on the physical origin of the halogen bond in terms of traditional VB concepts/descriptors. However, given the success of the present study in confirming the validity of well-established models for the $X B$ interaction, we expect that this can be the starting point for future and more quantitative investigations on halogen bonding through other Valence Bond techniques able to capture dynamic correlation effects, which are crucial to correctly take into account dispersion effects.

\section{Funding Information}

A.G. gratefully acknowledges the French Research Agency (ANR) for financial support through Grant No. ANR-17-CE29-0005.

The Fondazione Banca del Monte di Lombardia is also fully acknowledged for financial support.

\section{Authors Contribution}

A.G., M.S. and S.P. took care of conceptualization, formal analysis, writing original draft and review of the manuscript, and funding acquisition.

D.F. took care of data curation, formal analysis, visualization of figures, and writing the original draft of the manuscript.

F.D. contributed to the final review of the original manuscript and took care of visualization of some figures in the supplementary information.

Keywords: halogen bond, spin-coupled, valence bond, o-hole

(Additional Supporting Information may be found in the online version of this article)

\section{References and Notes}

1. P. Metrangolo, G. Resnati, Science 2008, $321,918$.

2. P. Metrangolo, G. Resnati, Halogen Bonding: Fundamentals and applications; Springer: berlin, 2008.

3. G. Cavallo, P. Metangolo, R. Milani, T. Pilati, A. Priimagi, G. Resnati, G. Terraneo, Chem. Rev. 2016, 116, 24782601.

4. Y. Lu, Y. Wang, W. Zhu, Phys. Chem. Chem. Phys. 2010, 12, 4543-4551.

5. P. Auffinger, F. A. Hays, E. Westhof, P. S. Ho, PNAS 2004, 101 (48), 16789-16794.

6. A. R. Voth, F. A. Hays, P. S. Ho, PNAS 2007, 104 (15), 6188-6193. 
7. E. Cariati, A. Forni, S. Biella, P. Metrangolo, F. Meyer, G. Resnati, S. Righetto, E. Tordin, R. Ugo, Chem. Commun. 2007, 0, 2590-2592.

8. E. Cariati, G. Cavallo, A. Forni, G. Leem, P. Metrangolo, F. Meyer, T. Pilati, G. Resnati, S. Righetto, G. Terraneo, E. Tordin, Cryst. Growth Des. 2011, 11 (12), 5642-5648.

9. P. K. Thallapally, G. R. Desiraju, M. Bagieu-Beucher, R. Masse, C. Bourgogne, J. Nicoud, Chem. Commun. 2002, 1052-1053.

10. T. M. Beale, M. G. Chudzinski, M. G. Sarwar, M. S. Taylor, Chem. Soc. Rev. 2013, 42 (4), 1667-1680.

11. A. Mele, P. Metrangolo, H. Neukirch, T. Pilati, G. Resnati, J. Am. Chem. Soc. 2005, 127 (43), 14972-14973.

12. D. Mango, G. Barbato, S. Piccirilli, M. B. Panico, M. Feligioni, C. Schepisi, M. Graziani, V. Porrini, M. Benarese, A. Lanzillotta, M. Pizzi, S. Pieraccini, M. Sironi, F. Blandini, F. Nicoletti, N. B. Mercuri, B. P. Imbimbo, R. Nisticò, Pharmacol. Res. 2014, 81, 83-90.

13. A. Priimagi, G. Cavallo, P. Metrangolo, G. Resnati, Acc. Chem. Res. 2013, 46 (11), 2686-2965.

14. P. Metrangolo, G. Resnati, Chem. Europ. J. 2001, 7 (12), 2511-2519.

15. S. Rendine, S. Pieraccini, A. Forni, M. Sironi, Phys. Chem. Chem. Phys. 2011, 13 (43), 19508-19516.

16. M. A. A. Ibrahim, J. Comput. Chem. 2011, 32 (12), 2564-2574.

17. T. Clark, M. Hennemann, J. S. Murray, P. Politzer, J. Mol. Mod. 2007, 13, 291-296.

18. K. Eskandari, H. Zariny, Chem. Phys. Lett. 2010, 491, 9-13.

19. R. F. W. Bader, Atoms in Molecules: A Quantum Theory; Oxford University Press: Oxford, U.K., 1990.

20. A. Forni, J. Phys. Chem. A 2009, 113 (14), 3403-3412.

21. V. Tognetti, L. Joubert Theor. Chem. Acc. 2015, 134, 90.
22. W. Changwei, D. Danovich, Y. Mo, S. J. Shaik, Chem. Theory Comput. 2014, 10, 3726-3737.

23. S. F. Boys, Rev. Mod. Phys. 1960, 32, 296-299.

24. J. M. Foster, S. F. Boys, Rev. Mod. Phys. 1960, 32, 300-302.

25. C. Edmiston, K. Ruedenberg, Rev. Mod. Phys. 1963, 35, 457-465.

26. C. Edmiston, K. J. Ruedenberg, Chem. Phys. 1965, 43, S97-S116.

27. J. Pipek, P. G. J. Mezey, Chem. Phys. 1989, 90, 4916-4926.

28. R. McWeeny, Proc. R. Soc. London Ser. A 1959, 253, 242-259.

29. R. McWeeny, Rev. Mod. Phys. 1960, 32, 335-369.

30. R. McWeeny, In Methods of Molecular Quantum Mechanics, 2nd ed.; Academic Press: London, 1992. Chapter 14, pp 485-495.

31. W. H. Adams, J. Chem. Phys. 1961, 34, 89-102.

32. S. Huzinaga, A. A. Cantu, J. Chem. Phys. $197155,5543-5549$.

33. T. L. Gilbert, J. Chem. Phys. 1974, 60, 3835-3844.

34. O. Matsuoka, J. Chem. Phys. 1977, 66, 1245-1254.

35. H. Stoll, G. Wagenblast, H. Preuss, Theor. Chim. Acta 1980, 57, 169-178.

36. G. F. Smits, C. Altona, Theor. Chim. Acta 1985, 67, 461-475.

37. E. Francisco, A. Martín Pendás, W. H. Adams, J. Chem. Phys. 1992, 97, 65046508.

38. P. Ordejón, D. Drabold, M. Grumbach, R. Martin, R. Phys. Rev. B 1993, 48, 14646-14649.

39. M. M. Couty, A. A. Bayse, M. B. Hall, Theor. Chem. Acc. 1997, 97, 96-109.

40. A. Fornili, M. Sironi, M. Raimondi, J. Mol. Struct. (THEOCHEM) 2003, 632, 157-172.

41. Z. Szekeres, P. R. Surján, Chem. Phys. Lett. 2003, 369, 125-130. 
42. M. Sironi, A. Genoni, M. Civera, S. Pieraccini, M. Ghitti, Theor. Chem. Acc. 2007, 117, 685-698.

43. A. Genoni, J. Phys. Chem. Lett. 2013, 4, 1093-1099.

44. A. Genoni, J. Chem. Theory Comput. 2013, 9, 3004-3019.

45. L. H. R. Dos Santos, A. Genoni, P. Macchi, Acta Crystallogr., Sect. A 2014, 70, 532-551.

46. A. Genoni, B. Meyer, Adv. Quantum Chem. 2016, 73, 333-362.

47. A. Fornili, M. Sironi, M. Raimondi, J. Mol. Struct. (THEOCHEM) 2003, 632, 157-172.

48. M. Sironi, A. Genoni, M. Civera, S. Pieraccini, M. Ghitti, Theor. Chem. Acc. 2007, 117, 685-698.

49. M. Sironi, A. Famulari, M. Raimondi, S. Chiesa, J. Mol. Struct. (THEOCHEM) 2000, 529, 47-54.

50. M. Sironi, M. Ghitti, A. Genoni, G. Saladino, S. Pieraccini, J. Mol. Struct. (THEOCHEM) 2009, 898, 8-16.

51. B. Meyer, B. Guillot, M. F. Ruiz-Lopez, A. Genoni, J. Chem. Theory Comput. 2016, 12, 1052-1067.

52. B. Meyer, B. Guillot, M. F. Ruiz-Lopez, A. Genoni, J. Chem. Theory Comput. 2016, 12, 1068-1081.

53. B. Meyer, A. Genoni, J. Phys. Chem. A 2018, 122, 8965-8981.

54. A. Genoni, M. Sironi, Theor. Chem. Acc. 2004, 112, 254-262.

55. A. Genoni, A. Fornili, M. Sironi, J. Comput. Chem. 2005, 26, 827-835.

56. A. Genoni, Acta Crystallogr., Sect. A 2017, 73, 312-316.

57. N. Casati, A. Genoni, B. Meyer, A. Krawczuk, P. Macchi, Acta Crystallogr., Sect. B 2017, 73, 584-597.

58. L. J. McAllister, D. W. Bruce, P. B. Karadakov, J. Phys. Chem. A 2012, 116, 10621-10628.

59. J. Gerratt, W. N. Lipscomb, Proc. Natl. Acad. Sci. U.S.A. 1968, 59, 332-335.
60. P. B. Karadakov, J. Gerratt, D. L. Cooper, M. Raimondi, J. Chem Phys. 1992, 97, 7637-7655.

61. D. L. Cooper, J. Gerratt, M. Raimondi, M. Sironi, T. Thorsteinsson, Theoret. Chim. Acta 1993, 85, 261-270.

62. D. Jayatilaka, Phys. Rev. Lett. 1998, 80, 798-801.

63. D. Jayatilaka, D. J. Grimwood, Acta Cryst. A 2001, 57, 76-86.

64. A. Genoni, L. Bučinský, N. Claiser, J. Contreras-García, B. Dittrich, P. M. Dominiak, E. Espinosa, C. Gatti, P. Giannozzi, J.-M. Gillet, D. Jayatilaka, P. Macchi, A. Ø. Madsen, L. J. Massa, C. F. Matta, K. M. Merz Jr., P. N. H. Nakashima, H. Ott, U. Ryde, K. Schwarz, M. Sierka, S. Grabowsky, Chem. Eur. J. 2018, 24, 10881-10905.

65. S. Grabowsky, A. Genoni, H.-B. Bürgi, Chem. Sci. 2017, 8, 4159-4176.

66. A. Genoni, D. Franchini, S. Pieraccini, M. Sironi, Chem. Eur. J. 2018, 24, 1550715511.

67. A. Genoni, L. H. R. Dos Santos, B. Meyer, P. Macchi, IUCrJ 2017, 4, 136-146.

68. In most of the situations it is sufficient to consider $\mathrm{S}=\mathrm{M}$. In the spin-coupled calculations for the present investigation we have always considered $\mathrm{S}=\mathrm{M}=0$.

69. B. H. Chirgwin, C. A. Coulson, VI. Proc. R. Soc. London, Ser. A 1950, 201, 196-209.

70. R. Ditchfield, W. J. Hehre, and J. A. Pople,,J. Chem. Phys. 1971, 54, 724728.

71. G. Rumer, Göttinger Nachr. 1932, 3, 337-341.

72. M. Simonetta, E. Gianinetti, I. Vandoni, J. Chem. Phys. 1968, 48, 1579-1594.

73. M. J. Frisch, G. W. Trucks, H. B. Schlegel, G. E. Scuseria, M. A. Robb, J. R.

Cheeseman, G. Scalmani, V. Barone, B. Mennucci, G. A. Petersson, H. Nakatsuji, M. Caricato, X. Li, H. P. Hratchian, A. F. Izmaylov, J. Bloino, G. Zheng, J. L. Sonnenberg, M. Hada, M. Ehara, K. Toyota, R. Fukuda, J. Hasegawa, M. 
Morokuma, V. G. Zakrzewski, G. A.

Ishida, T. Nakajima, Y. Honda, O. Kitao, H. Nakai, T. Vreven, J. A. Montgomery, Jr., J. E. Peralta, F. Ogliaro, M. Bearpark, J. J. Heyd, E. Brothers, K. N. Kudin, V. N. Staroverov, R. Kobayashi, J. Normand, K. Raghavachari, A. Rendell, J. C. Burant, S. S. lyengar, J. Tomasi, M. Cossi, N. Rega, J. M. Millam, M. Klene, J. E. Knox, J. B. Cross, V. Bakken, C. Adamo, J. Jaramillo, R. Gomperts, R. E. Stratmann, O. Yazyev, A. J. Austin, R. Cammi, C. Pomelli, J. W. Ochterski, R. L. Martin, K.
Voth, P. Salvador, J. J. Dannenberg, S. Dapprich, A. D. Daniels, Ö. Farkas, J. B. Foresman, J. V. Ortiz, J. Cioslowski, and D. J. Fox, Gaussian 09 (Gaussian, Inc., Wallingford CT, 2009).

74. D. L. Cooper, J. Gerratt, M. Raimondi, M. Sironi, T. Thorsteinsson, Theoret. Chim. Acta 1993, 85, 261. 
Davide Franchini, Alessandro Genoni, Federico Dapiaggi, Stefano Pieraccini, and Maurizio Sironi

\section{A valence bond description of the bromine halogen bond}

Halogen bond is an interesting type of non-covalent interaction that is drawing lot of attention due to its possible application in several fields. In this paper, the nature of this interaction has been investigated using a Valence Bond approach. This enabled not only to confirm the main features of the previously proposed models (e.g., Politzer model), but also to shed further light on the physics of halogen bond, by introducing the new concept of $\sigma$-tunnel.

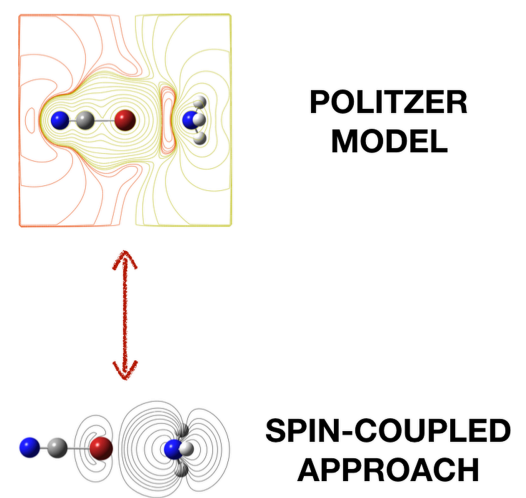

